\title{
The effect of lymph node dissection on cancer-specific survival in salvage radical prostatectomy patients
}

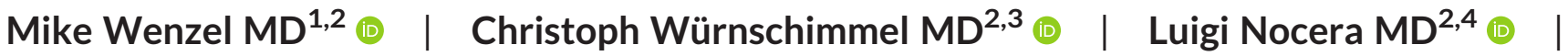 \\ Claudia Collà Ruvolo MD ${ }^{2,5}$ @ | Zhe Tian $\mathrm{MSc}^{2}$ | \\ Shahrokh F. Shariat MD, PhD $6,7,8,9,10,11$ | Fred Saad MD, PhD $^{2}$ | \\ Alberto Briganti MD, $\mathrm{PhD}^{4} \quad$ Markus Graefen MD, $\mathrm{PhD}^{3} \quad$ Luis A. Kluth MD, $\mathrm{PhD}^{1}$ | \\ Philipp Mandel MD, PhD ${ }^{1}$ | Felix K. H. Chun MD, $\mathrm{PhD}^{1}$ \\ Pierre I. Karakiewicz MD, $\mathrm{PhD}^{2}$
}

\begin{abstract}
${ }^{1}$ Department of Urology, University Hospital Frankfurt, Goethe University Frankfurt, Frankfurt, Germany
${ }^{2}$ Cancer Prognostics and Health Outcomes Unit, Division of Urology, University of Montréal Health Center, Montréal, Québec, Canada

${ }^{3}$ Martini-Klinik Prostate Cancer Center, University Hospital Hamburg-Eppendorf, Hamburg, Germany

${ }^{4}$ Department of Urology and Division of Experimental Oncology, Urological Research Institute, IRCCS San Raffaele Scientific Institute, Milan, Italy

${ }^{5}$ Department of Neurosciences, Reproductive Sciences and Odontostomatology, University of Naples Federico II, Naples, Italy

${ }^{6}$ Department of Urology, Comprehensive Cancer Center, Medical University of Vienna, Vienna, Austria

${ }^{7}$ Departments of Urology, Weill Cornell Medical College, New York, New York, USA

${ }^{8}$ Department of Urology, University of Texas Southwestern, Dallas, Texas, USA

${ }^{9}$ Department of Urology, Second Faculty of Medicine, Charles University, Prag, Czech Republic

${ }^{10}$ Department of Urology, Institute for Urology and Reproductive Health, I.M. Sechenov First Moscow State Medical University, Moscow, Russia

${ }^{11}$ Division of Urology, Department of Special Surgery, Jordan University Hospital, The University of Jordan, Amman, Jordan
\end{abstract}

\section{Correspondence}

Mike Wenzel, MD, Department of Urology, University Hospital Frankfurt, Goethe University Frankfurt, Theodor- Stern Kai 7, 60590 Frankfurt, Germany.

Email: Mike.Wenzel@kgu.de

\begin{abstract}
Background: We hypothesized that lymph node dissection (LND) at salvage radical prostatectomy may be associated with lower cancer-specific mortality (CSM) and we tested this hypothesis.

Methods: We relied on surveillance, epidemiology, and end results (2004-2016) to identify all salvage radical prostatectomy patients. Categorical, as well as univariate and multivariate Cox regression models tested the effect of LND (LND performed vs. not), as well as at its extent (log-transformed lymph node count) on CSM.

Results: Of 427 salvage radical prostatectomy patients, 120 (28.1\%) underwent LND with a median lymph node count of 6 (interquartile range [IQR], 3-11). According to LND status, no significant or clinically meaningful differences were recorded in PSA at diagnosis, stage and biopsy Gleason score at diagnosis, except for age at prostate cancer diagnosis (LND performed 63 vs. 68 years LND not
\end{abstract}


performed, $p<.001$ ). LND status (performed) was an independent predictor of lower CSM (hazard ratio [HR] 0.47; $p=.03$ ). Similarly, lymph node count (log transformed) also independently predicted lower CSM (HR: 0.60; $p=.01$ ). After the 7th removed lymph node, the effect of CSM became marginal. The effect of $\mathrm{N}$-stage on CSM could not be tested due to insufficient number of observations.

Conclusions: Salvage radical prostatectomy is rarely performed and LND at salvage radical prostatectomy is performed in a minority of patients. However, LND at salvage radical prostatectomy is associated with lower CSM. Moreover, LND extent also exerts a protective effect on CSM. These observations should be considered in salvage radical prostatectomy candidates.

\section{KEYWORDS}

cancer specific survival, lymph node dissection, prostate cancer, salvage radical prostatectomy

\section{1 | INTRODUCTION}

Salvage radical prostatectomy represents a treatment option for patients with radio-recurrent prostate cancer. ${ }^{1-6}$ Lymph node dissection (LND) should ideally be performed at salvage radical prostatectomy. To the best of our knowledge, only one large historical study ( $n=364$, 1988-2010) tested the effect of LND on cancer-specific mortality (CSM) at salvage radical prostatectomy. ${ }^{7}$ We hypothesized that LND at salvage radical prostatectomy may be associated with lower CSM and we tested this hypothesis in a contemporary population-based cohort (surveillance, epidemiology and end results [SEER] database 2004-2016).

\section{2 | MATERIAL AND METHODS}

\section{1 | Study population}

The current SEER database samples $34.6 \%$ of the United States population and approximates it in demographic composition and cancer incidence. ${ }^{8}$ Within the SEER database (2004-2016), we identified patients $\geq 18$ years old with histologically confirmed adenocarcinoma of the prostate, diagnosed at biopsy (International Classification of Disease for Oncology [ICD-O-3] code 8140 site code C61.9). Cases identified at autopsy or through death certificates, with unknown histology, nonprimary prostate cancers, or patients with unknown LND status were excluded. Salvage radical prostatectomy was defined as prostatectomy after radiation therapy, as previously described. ${ }^{7}$ PSA and age and stage were defined at initial prostate cancer diagnosis. These selection criteria resulted in a cohort of 427 salvage radical prostatectomy patients.

\section{2 | Statistical analysis}

Descriptive statistics included frequencies and proportions for categorical variables. Means, medians, and interquartile-ranges (IQR) were reported for continuously coded variables. The chi-square tested the statistical significance in proportions' differences. The $t$ test and Kruskal-Wallis test examined the statistical significance of means' and distributions' differences.

Kaplan-Meier, univariate and multivariate Cox regression models tested the effect of LND on CSM in salvage radical prostatectomy patients. Moreover, separate models tested the effect of log-transformed continuously coded lymph node count on CSM. All tests were two-sided with a level of significance set at $p<.05$ and $R$ software environment for statistical computing and graphics (version 3.4.3) was used for all analyses. ${ }^{9}$

\section{3 | RESULTS}

\subsection{Descriptive characteristics of the study population}

Of 427 salvage radical prostatectomy patients (Table 1), 120 (28.1\%) underwent LND. The median age at diagnosis was 63 years (IQR, 58-67) in LND versus 68 years (IQR, 62-75) in no LND patients. Moreover, median PSA at diagnosis was $8.9 \mathrm{ng} / \mathrm{ml}(5.6-14.6)$ in LND versus $8.7 \mathrm{ng} / \mathrm{ml}$ (IQR, 5.4-24.0) in no LND patients $(p=.3)$. No differences according to LND status were recorded for Gleason score at biopsy $(p=.9)$ or clinical T stage at diagnosis $(p=.8)$. Moreover, no differences were recorded according to a previous type of radiation therapy (external beam radiation therapy [EBRT] vs. brachytherapy $[\mathrm{BT}]$ vs. EBRT+BT) between both groups $(p=.9)$. Radical prostatectomy Gleason scores could not be analyzed, due to large proportions of missing values (LND performed: $73.3 \%$ missing vs. $94.1 \%$ missing when LND not performed). Similar limitations applied to pathological T stage (LND performed: $23.3 \%$ missing vs. $81.4 \%$ missing when LND not performed). Follow-up duration was 71 (IQR, 29-108) months in no LND versus $89(40-126)$ months in the LND group $(p=.02)$. 
TABLE 1 Descriptive characteristics of 427 salvage radical prostatectomy patients, stratified according to lymph node dissection status, diagnosed within the Surveillance, Epidemiology, and End Results database from 2004 to 2016

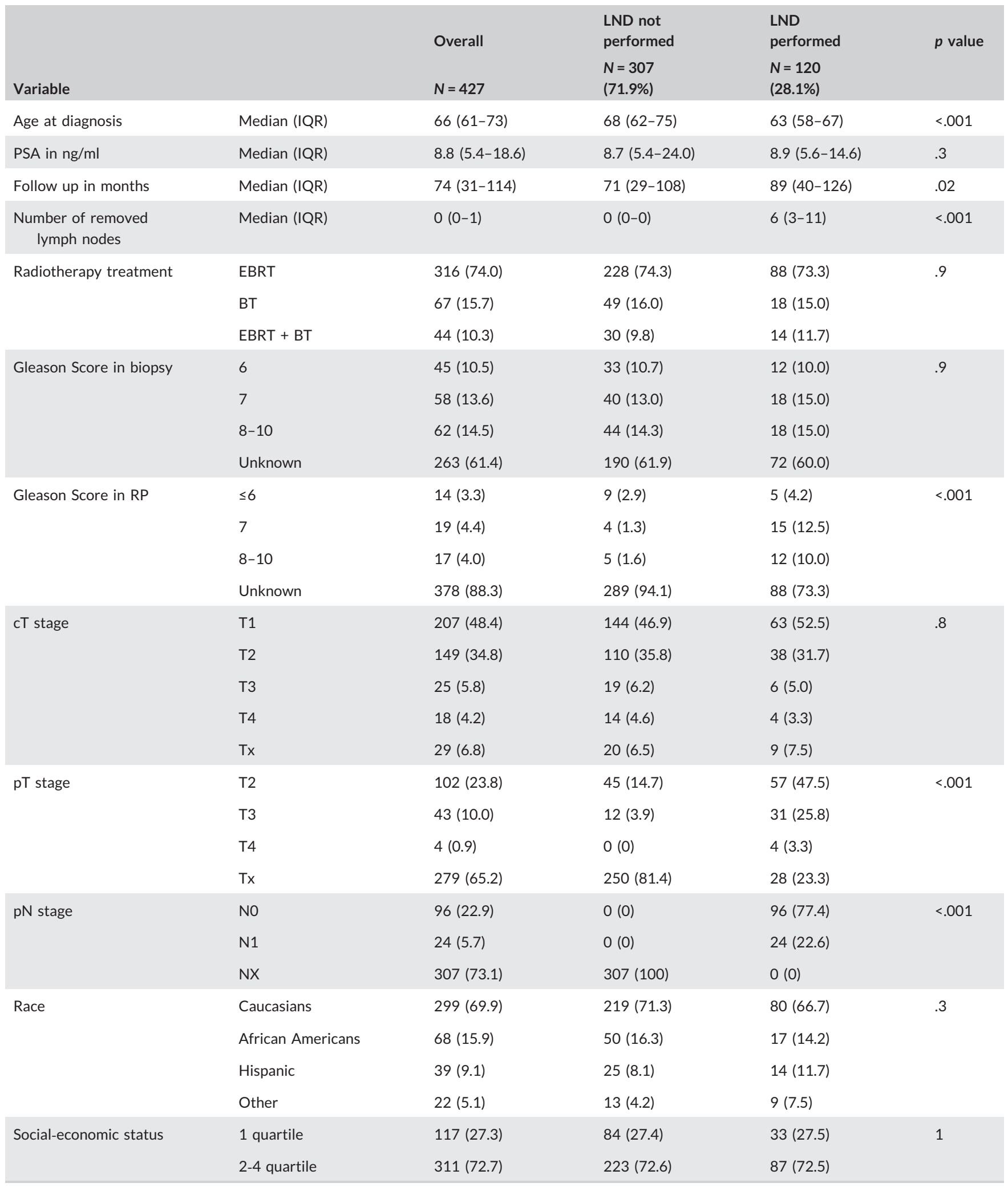

Abbreviations: BT, brachytherapy; EBRT, external beam radiation therapy; IQR, interquartile range; LND, lymph node dissection; PSA, prostate-specific antigen; RP, radical prostatectomy. 


\section{CSM in Salvage Radical Prostatectomy - LND not performed - LND performed}

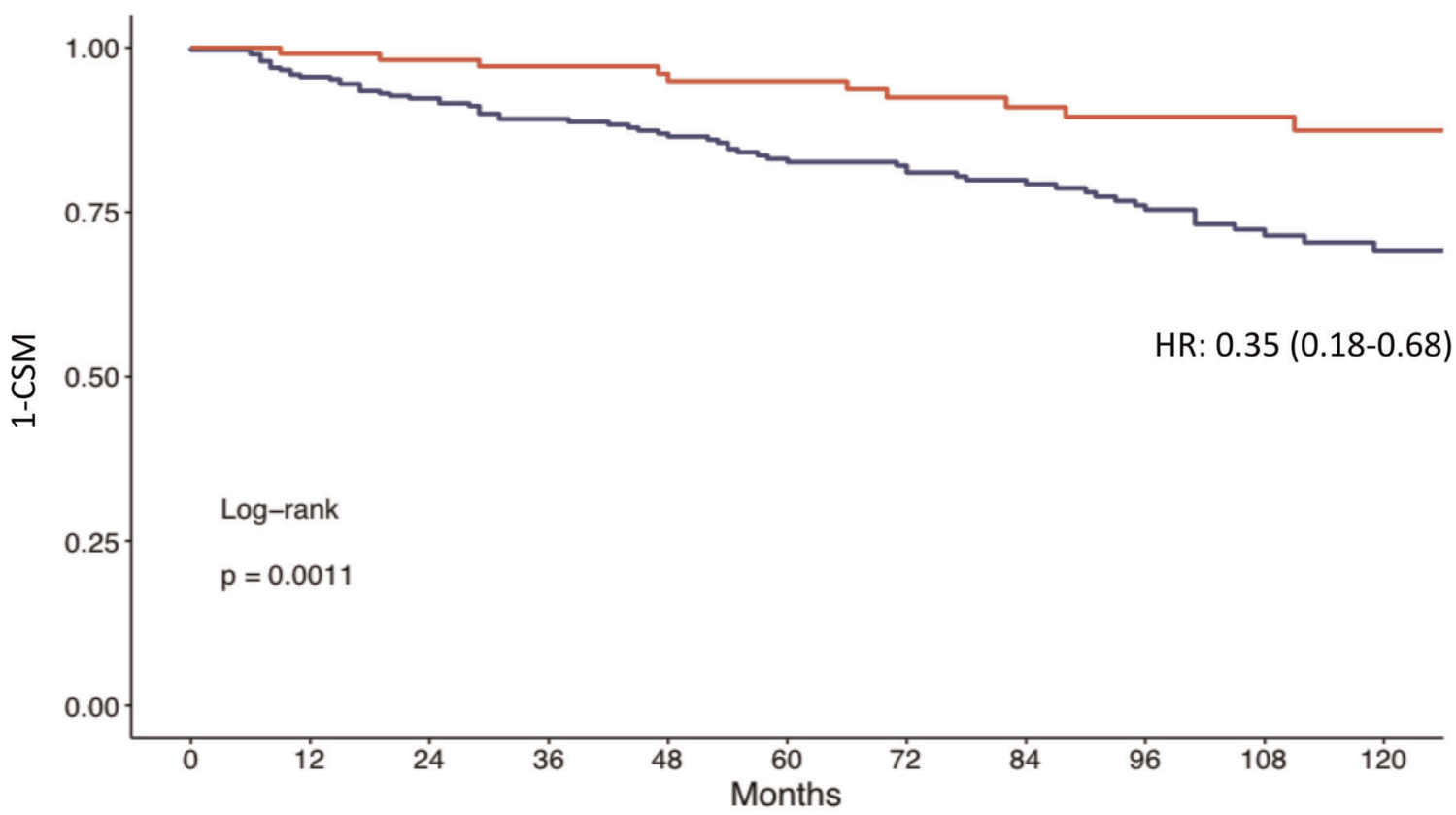

Number at risk

\begin{tabular}{|c|c|c|c|c|c|c|c|c|c|c|c|}
\hline LND not performed & 307 & 269 & 243 & 216 & 187 & 167 & 153 & 132 & 111 & 77 & 57 \\
\hline \multirow[t]{2}{*}{ LND performed } & 120 & 108 & 100 & 94 & 86 & 82 & 69 & 61 & 54 & 45 & 35 \\
\hline & 0 & 12 & 24 & 36 & 48 & $\begin{array}{c}60 \\
\text { Month }\end{array}$ & 72 & 84 & 96 & 108 & 120 \\
\hline
\end{tabular}

FIGURE 1 Kaplan-Meier plot illustrating cancer-specific mortality (CSM) for patients, who underwent salvage radical prostatectomy, stratified according to the performance of lymph node dissection (LND) status. Abbreviations: HR, hazard ratio [Color figure can be viewed at wileyonlinelibrary.com]

TABLE 2 Univariable and multivariable Cox regression models in salvage radical prostatectomy patients predicting cancer-specific mortality

\begin{tabular}{|c|c|c|c|c|c|c|}
\hline & \multicolumn{3}{|c|}{ Univariate analysis } & \multicolumn{3}{|c|}{ Multivariate analysis } \\
\hline & HR & $95 \% \mathrm{Cl}$ & $p$ value & HR & $95 \% \mathrm{Cl}$ & $p$ value \\
\hline Age & 1.01 & $(0.99-1.04)$ & 0.3 & 1.01 & $(0.98-1.04)$ & .6 \\
\hline LND not performed & 1 (Ref) & - & - & 1 (Ref) & - & - \\
\hline LND performed & 0.35 & $(0.18-0.68)$ & $<0.01$ & 0.46 & $(0.23-0.92)$ & .03 \\
\hline Gleason score biopsy 7 & 4.33 & $(0.48-38.81)$ & 0.19 & 2.79 & $(0.30-25.60)$ & .4 \\
\hline Gleason score biopsy 8-10 & 17.85 & $(2.35-135.32)$ & $<0.01$ & 5.51 & $(0.69-43.94)$ & .11 \\
\hline Gleason score biopsy unknown & 5.97 & $(0.82-43.36)$ & 0.077 & 2.76 & $(0.37-20.61)$ & .3 \\
\hline BT & 1 (Ref) & - & - & 1 (Ref) & - & - \\
\hline EBRT & 3.01 & $1.30-6.96$ & 0.01 & 1.42 & $0.59-3.44$ & .4 \\
\hline $\mathrm{EBRT}+\mathrm{BT}$ & 1.23 & $0.38-4.04$ & 0.7 & 0.74 & $0.22-2.51$ & .6 \\
\hline
\end{tabular}

Abbreviations: BT, brachytherapy; EBRT, external beam radiation therapy; LN, lymph node. 


\subsection{The effect of LND on CSM status at salvage radical prostatectomy}

In the first part of the analyses, we tested the effect of LND status (performed vs. not performed) on CSM (Figure 1). Specifically, in Kaplan-Meier plots, salvage radical prostatectomy patients treated with LND exhibited lower CSM than no LND (hazard ratio [HR]: 0.35, confidence interval $[\mathrm{Cl}]: 0.18-0.68 ; p=.001)$. At 10 years, CSM was $12.6 \%$ for LND versus $30.8 \%$ for no LND. After multivariate adjustment (Table 2) for age, PSA, Gleason score at biopsy and clinical T-stage at diagnosis and initial radiation treatment type, LND was an independent predictor of lower CSM (HR 0.46, CI: 0.23-0.92; $p=.03$ ).

\subsection{The effect of LND extent on CSM at salvage radical prostatectomy}

First, median number of removed lymph nodes at salvage radical prostatectomy patients was 6 (IQR, 3-11). Lymph node invasion was identified in 24 ( $n=22.6 \%$ ) patients. Left skewed lymph node count distribution (skewness, 3.17) was log-transformed to arrive at more normally distributed values (skewness, 1.51) of lymph node count. In univariate Cox regression models (Table 3), increasing values of logtransformed lymph node count was associated with lower CSM (HR: 0.53, Cl: 0.36-0.79; $p$ <01). Moreover, in multivariate Cox regression models, increasing lymph node count was associated with lower CSM (HR: $0.60, \mathrm{Cl}$ : 0.40-0.90; $p=.01$ ). Graphical representation of the relationship between log-transformed lymph node count versus CSM clearly showed an inverse relationship (Figure 2). This relationship translated to a CSM decrease of respectively $30 \%, 43 \%$, $51 \%, 56 \%, 60 \%, 63 \%, 65 \%, 67 \%, 69 \%, 71 \%$, and $79 \%$ for the 1 st, 2 nd, 3 rd, 4th, 5th, 6th, 7th, 8th, 9th, 10th, 20th removed lymph node. Thus, the estimated reduction in hazard of CSM for the removal of the first lymph node is $30 \%$, but to further reduce the hazard to the same magnitude, five lymph nodes need to be removed, and the effect of removing more lymph nodes becomes almost negligible after 7th lymph node. The change in CSM with each additional removed lymph node was $<1 \%$ after the 13 th lymph node.

\section{4 | DISCUSSION}

We hypothesized that LND at salvage radical prostatectomy may be associated with lower CSM. We tested this concept in a contemporary cohort of salvage radical prostatectomy patients and made several noteworthy observations.

First, within the entire SEER database 2004-2016 we only identified 427 salvage radical prostatectomy patients. This small number sharply contrasts with other SEER-based studies that addressed primary radical prostatectomy patients with counts of over 150,000 patients. ${ }^{10}$ This observation indicates that salvage radical prostatectomy is performed exceedingly rarely. This fact is consistent with institutional series, where a very small fraction of all radical prostatectomy patients are treated in a salvage context. $^{3,4,6,11-14}$ For example, in the study of Sanderson et al. of 2739 radical prostatectomy patients (1983-2002), only 51 were treated in the salvage radical prostatectomy context (6). Moreover, of 430 radiotherapy-recurrent patients in the CaPSURE database, only four

TAB LE 3 Univariate and multivariate Cox regression models after log transformation for count of removed lymph nodes in salvage radical prostatectomy patients predicting cancer-specific mortality (CSM)

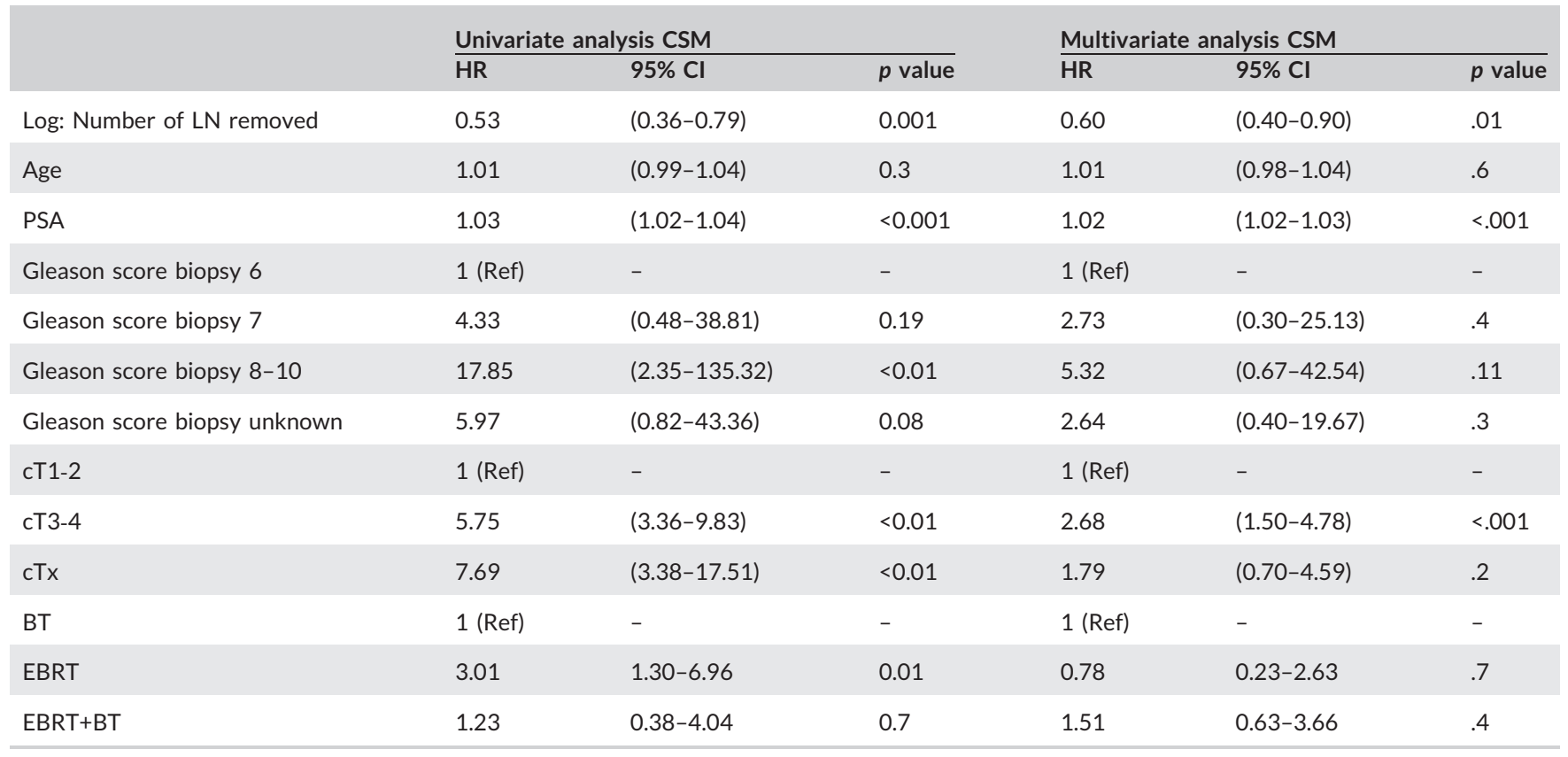

Abbreviations: BT, brachytherapy; EBRT, external beam radiation therapy; LN, lymph node. 


\section{Correlation between lymph node count and CSM-free survival in SRP}

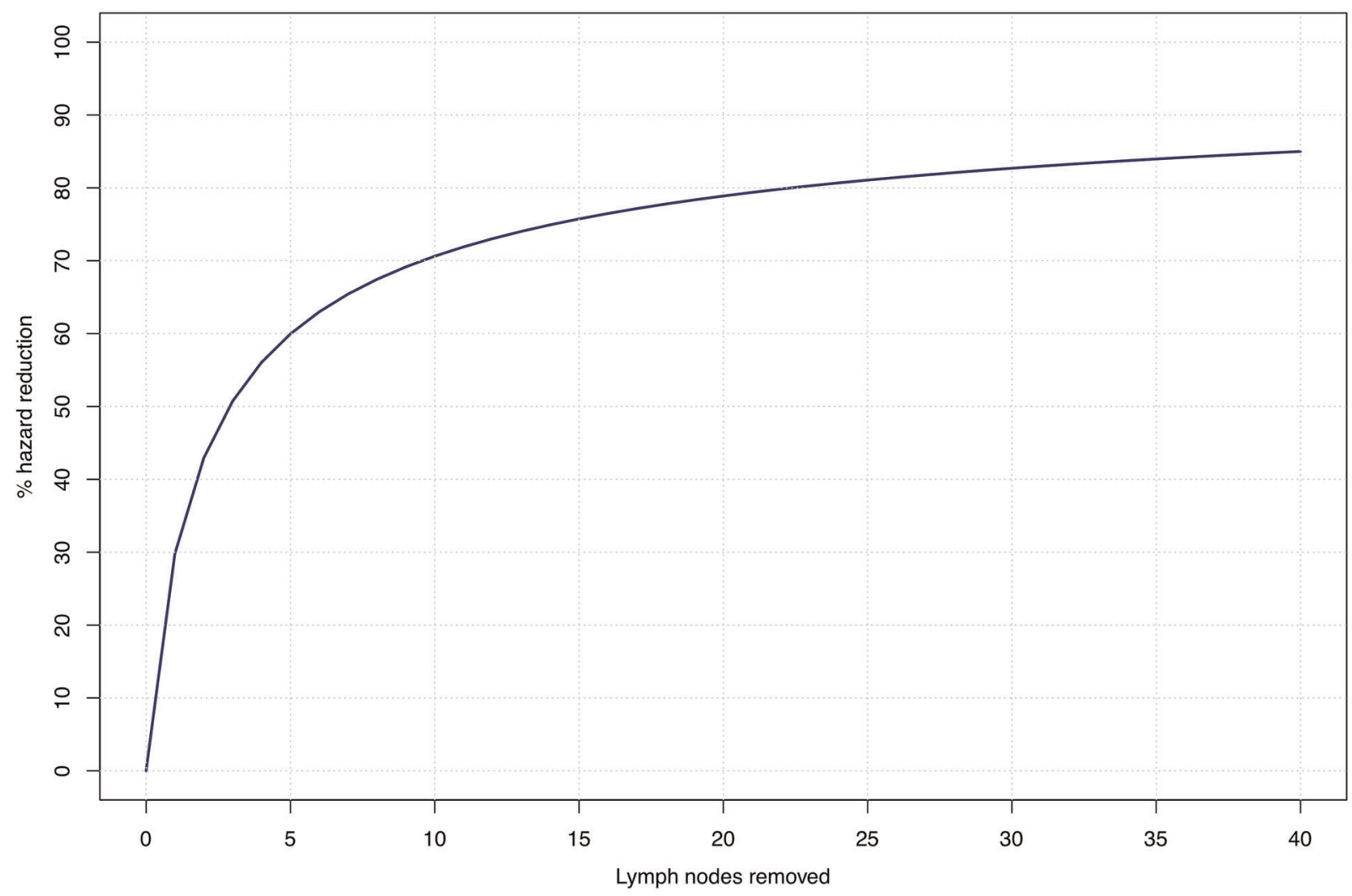

FIGURE 2 Correlation between lymph node count at lymph node dissection (LND) at salvage radical prostatectomy and percentage of cancer-specific mortality (CSM)-free survival after log transformation [Color figure can be viewed at wileyonlinelibrary.com]

patients $(0.9 \%)$ underwent salvage radical prostatectomy. ${ }^{15}$ Despite the rarity of this procedure and the highly select nature of salvage radical prostatectomy patients, we identified strong and meaningful associations that pertained to LND status (performed vs not performed), as well as to LND extent.

Regarding LND status, LND at salvage radical prostatectomy was associated with lower CSM. This association was equally strong before (HR: $0.35, \mathrm{Cl}: 0.18-0.68 ; p<.01$ ), as well as after multivariate adjustment for patient and tumor characteristics (HR: $0.46, \mathrm{Cl}$ : $0.23-0.92 ; p=.03$ ). The magnitude of CSM differences at ten years may be illustrated with $12.6 \%$ CSM after LND versus $30.8 \%$, when LND was not performed. The above results illustrate the effect of LND in most contemporary salvage radical prostatectomy setting, and they do agree with more historical figures. For example, Pokala et al. ( $n=364,1988-2010)$ reported a decrease in CSM with LND at salvage radical prostatectomy. However, its magnitude was lower (univariate $p=.14$, multivariate: 2.7 -fold higher CSM without LND, $p=.01$ ). Greater benefit of LND in contemporary patients may be related to differences in LND extent between the historical study of Pokala et al. and the current data. In the historical era that was described by Pokala et al., either no LND or very limited LND was usually performed at radical prostatectomy in general. ${ }^{16}$ For example, Doublet et al. $^{16}$ reported an average of 8 removed lymph nodes in their study, covering the years 1991-1993. This value sharply contrasts with contemporary numbers of removed lymph nodes, that range from 14 to $16 .^{17,18}$

An alternative explanation for the difference in the magnitude of CSM protective effect may be provided. For example, it may be postulated that all newly diagnosed historical patients harbored more important disease burden (e.g., higher $\mathrm{T}$ stage), relative to contemporary patients. ${ }^{19}$ In consequence, LND may have had a more limited effect on CSM than in contemporary patients, in whom stage migration has resulted in significantly lower disease burden. However, formal comparisons between historical and contemporary LND at salvage radical prostatectomy cannot be made.

Regarding LND extent, we also identified an association, whereby higher number of removed lymph nodes was associated with lower CSM. Specifically, the highest effect on CSM was observed for the first seven lymph nodes. After the 13th removed lymph node, the effect on CSM became $<1 \%$ for each additional removed lymph node. The concept of the lymph node extent on CSM has been previously described in primary radical prostatectomy series. ${ }^{20,21}$ Unfortunately, only a few studies examined this association in a controlled fashion and compared standard versus extended LND. Specifically, Heidenreich et al. ${ }^{22}$ and Clarke et al. ${ }^{23}$ performed controlled (randomized) studies of standard versus extended LND. However, these analyses relied on limited sample sizes $(n=203$ and $n=123$ ). Moreover, these studies were not designed to address the 
effect of lymph node count defined as number of removed lymph nodes on CSM at radical prostatectomy. Our study is not an exception to the limitations in sample size regarding the effect of LND on CSM. In consequence, our study could not provide more detailed analyses such as those relying on pathological N1 versus NO versus $\mathrm{Nx}$ status. Additionally, relative to conventional radical prostatectomy series, our observed lymph node count in the current cohort may be interpreted as suboptimal and more efforts should be made on the extent of LND at salvage radical prostatectomy.

Additionally, we observed lack of statistically or clinically meaningful differences in PSA, clinical stage and Gleason score at biopsy according to LND status (performed vs. no LND). These observations suggest that patient characteristics at initial prostate cancer diagnosis did not affect LND rates at salvage radical prostatectomy. However, based on unavailable information about longitudinal PSA values since diagnosis, we could not test the effect of PSA values before salvage radical prostatectomy on either LND status or on CSM. It should be emphasized that such values may be difficult to interpret due to androgen deprivation therapy (ADT) administration with radiotherapy. It is also particularly noteworthy that a large proportion of patients also had unavailable pathological stage and Gleason scores at salvage radical prostatectomy. Similar limitations apply to clinical $\mathrm{T}$ stage and Gleason score after failed radiotherapy and before salvage radical prostatectomy. Here, the same considerations apply as for PSA values. Specifically, exposure to ADT renders stage and grade assignment is challenging and stage and grade are not invariably reported. ${ }^{5,24}$ It is well-known that exposure to ADT before radical prostatectomy renders standard assessment of pathological stage and Gleason score and is challenging or even impossible without immunohistochemical staining. ${ }^{25}$

Despite the above limitations, as well as other limitations associated with retrospective series such as the current one, our observations are in accordance with established observations and their effect of the natural treated history of prostate cancer. Specifically, in general, LND is associated with lower CSM at primary radical prostatectomy. Similarly, at primary radical prostatectomy, more extensive LND is also associated with lower CSM. ${ }^{20,26}$ Nonetheless, further studies focusing on similar large-scaled national databases or in best case scenario prospective multi-institutional studies should ideally validate or refute our findings, since findings from populationbased studies may differ from nonpopulation-based studies. ${ }^{27}$ For example, the National Cancer Database provides a larger sample of prostate cancer patients than the SEER database. Unfortunately, the National Cancer Database structure does not provide CSM, instead only overall mortality is reported. All analyses that examined the effect of LND in the salvage, as well as primary setting relied on CSM. In consequence, overall mortality may not represent a sufficiently specific endpoint. National Cancer Database data may not represent an ideal choice, based in this consideration. However, large-scale databases, such as the most contemporary version of CaPSURE with a higher number of salvage radical prostatectomy patients, may provide CSM outcomes in addition to more detailed pathological specimen assessment of primary tumor and lymph nodes. Ideally, additional analyses should also address European patients to corroborate or refute the universality of our findings between continents.

\section{5 | CONCLUSION}

Salvage radical prostatectomy is rarely performed and LND at salvage radical prostatectomy is performed in a minority of patients. However, LND at salvage radical prostatectomy is associated with lower CSM. Moreover, LND extent also exerts a protective effect on CSM. These observations should be considered in salvage radical prostatectomy candidates.

\section{ACKNOWLEDGMENT}

Open Access funding enabled and organized by Projekt DEAL.

\section{FUNDING STATEMENT}

The research was conducted in the absence of any commercial or financial relationships that could be construed as a potential conflict of interest.

\section{CONFLICT OF INTERESTS}

The authors declare that there are no conflict of interests.

\section{DATA AVAILABILITY STATEMENT}

All data generated for this analysis were from the SEER 18 database. The code for the analyses will be made available after request.

\section{ETHICS CONSENT STATEMENT}

All analyses and their reporting followed the SEER reporting guidelines. Due to the anonymously coded design of the SEER database, study-specific Institutional Review Board ethics approval was not required.

\section{ORCID}

Mike Wenzel (D) http://orcid.org/0000-0002-4338-0889

Christoph Würnschimmel (D) http://orcid.org/0000-0001-7891-4791

Luigi Nocera (D) http://orcid.org/0000-0003-3354-8139

Claudia Collà Ruvolo (D) https://orcid.org/0000-0001-8110-7341

\section{REFERENCES}

1. Mottet N, Cornford P, Van den Bergh RCN, et al. EAU - EANM ESTRO - ESUR - SIOG. guidelines on prostate cancer. Eur Urol. 2020.

2. Mohler JL, Antonarakis ES, Armstrong AJ, et al. Prostate cancer, version 1.2020, NCCN clinical practice guidelines in oncology. J Natl Compr Canc Netw. 2020

3. Mandel P, Steuber T, Ahyai S, et al. Salvage radical prostatectomy for recurrent prostate cancer: verification of European Association of Urology guideline criteria. BJU Int. 2016;117(1):55-61.

4. Heidenreich A, Richter S, Thüer D, Pfister D. Prognostic parameters, complications, and oncologic and functional outcome of salvage radical prostatectomy for locally recurrent prostate cancer after 21st-century radiotherapy. Eur Urol. 2010;57(3): 437-443. 
5. Chade DC, Shariat SF, Cronin AM, et al. Salvage radical prostatectomy for radiation-recurrent prostate cancer: a multiinstitutional collaboration. Eur Urol. 2011;60(2):205-210.

6. Sanderson KM, Penson DF, Cai J, et al. Salvage radical prostatectomy: quality of life outcomes and long-term oncological control of radiorecurrent prostate cancer. J Urol. 2006;176(5):2025-2031.

7. Pokala N, Huynh DL, Henderson AA, Johans C. Survival outcomes in men undergoing radical prostatectomy after primary radiation treatment for adenocarcinoma of the prostate. Clin Genitourin Cancer. 2016;14(3):218-225.

8. About the SEER Program [Internet]. SEER. [cited 2020 August 20]. Available from: https://seer.cancer.gov/about/overview.html

9. RCT. R: A language and environment for statistical computing. https://wwwr-projectorg2017

10. Preisser F, Mazzone E, Knipper S, et al. Rates of positive surgical margins and their effect on cancer-specific mortality at radical prostatectomy for patients with clinically localized prostate cancer. Clin Genitourin Cancer. 2019;17(1):e130-e139.

11. Heidenreich A, Ohlmann C, Ozgür E, Engelmann U. Functional and oncological outcome of salvage prostatectomy of locally recurrent prostate cancer following radiation therapy. Urologe A. 2006;45(4):474-481.

12. Gheiler EL, Tefilli MV, Tiguert R, et al. Predictors for maximal outcome in patients undergoing salvage surgery for radio-recurrent prostate cancer. Urology. 1998;51(5):789-795.

13. Stephenson AJ, Scardino PT, Bianco FJ, DiBlasio CJ, Fearn PA, Eastham JA. Morbidity and functional outcomes of salvage radical prostatectomy for locally recurrent prostate cancer after radiation therapy. J Urol. 2004;172(6 Pt 1):2239-2243.

14. Ward JF, Sebo TJ, Blute ML, Zincke H. Salvage surgery for radiorecurrent prostate cancer: contemporary outcomes. J Urol. 2005; 173(4):1156-1160.

15. Agarwal PK, Sadetsky N, Konety BR, Resnick MI, Carroll PR. Cancer of the Prostate Strategic Urological Research Endeavor (CaPSURE). Treatment failure after primary and salvage therapy for prostate cancer: likelihood, patterns of care, and outcomes. Cancer. 2008; 112(2):307-314

16. Doublet JD, Gattegno B, Thibault P. Laparoscopic pelvic lymph node dissection for staging of prostatic cancer. Eur Urol. 1994;25(3):194-198.

17. Preisser F, van den Bergh RCN, Gandaglia G, et al. Effect of extended pelvic lymph node dissection on oncologic outcomes in patients with d'amico intermediate and high risk prostate cancer treated with radical prostatectomy: a multi-institutional study. J Urol. 2020;203(2): 338-343.

18. Gandaglia G, De Lorenzis E, Novara G, et al. Robot-assisted radical prostatectomy and extended pelvic lymph node dissection in patients with locally-advanced prostate cancer. Eur Urol. 2017;71(2): 249-256.

19. Hoffman RM, Meisner ALW, Arap W, et al. Trends in United States prostate cancer incidence rates by age and stage, 1995-2012. Cancer Epidemiol Biomarkers Prev. 2016;25(2):259-263.

20. Abdollah F, Gandaglia G, Suardi N, et al. More extensive pelvic lymph node dissection improves survival in patients with nodepositive prostate cancer. Eur Urol. 2015;67(2):212-219.

21. Moschini M, Fossati N, Abdollah F, et al. Determinants of long-term survival of patients with locally advanced prostate cancer: the role of extensive pelvic lymph node dissection. Prostate Cancer Prostatic Dis. 2016;19(1):63-67.

22. Heidenreich A, Varga Z, Von Knobloch R. Extended pelvic lymphadenectomy in patients undergoing radical prostatectomy: high incidence of lymph node metastasis. J Urol. 2002;167(4): 1681-1686.

23. Clark T, Parekh DJ, Cookson MS, et al. Randomized prospective evaluation of extended versus limited lymph node dissection in patients with clinically localized prostate cancer. J Urol. 2003, 169(1):145-147

24. Molinié V, Mahjoub WK, Balaton A. Histological modifications observed in prostate after preserving treatments for prostate cancer and their impact on Gleason score interpretation. Ann Pathol. 2008; 28(5):363-373.

25. Bazinet $M$, Zheng W, Bégin LR, Aprikian AG, Karakiewicz PI, Elhilali MM. Morphologic changes induced by neoadjuvant androgen ablation may result in underdetection of positive surgical margins and capsular involvement by prostatic adenocarcinoma. Urology. 1997;49(5):721-725.

26. Preisser F, Bandini M, Marchioni M, et al. Extent of lymph node dissection improves survival in prostate cancer patients treated with radical prostatectomy without lymph node invasion. Prostate. 2018;78(6):469-475.

27. Soni PD, Hartman HE, Dess RT, et al. Comparison of populationbased observational studies with randomized trials in oncology. J Clin Oncol Off J Am Soc Clin Oncol. 2019;37(14):1209-1216.

How to cite this article: Wenzel M, Würnschimmel C, Nocera L, et al. The effect of lymph node dissection on cancer-specific survival in salvage radical prostatectomy patients. The Prostate. 2021;81:339-346. https://doi.org/10.1002/pros.24112 